\author{
E-ISSN: 2469-6501 \\ VOL: 7, ISSUE: 8 \\ August/2021 \\ DOI: http://dx.doi.org/10.33642/ijbass.v7n8p7 \\ https://creativecommons.org/licenses/by/4.0/
}

\title{
The Use of Electronic Health Record Systems During COVID-19 at a Local Community Hospital on the West Coast
}

Malini Krishnamurthi, Ph.D.

Department of Information and Decision Sciences

California State University

Fullerton, California

Email: Mkrishnamurthi@fullerton.edu

United States of America

\section{ABSTRACT}

The United States Federal government looks toward information technology to curtail health care costs while increasing the quality of patient care through the adoption of electronic health record (EHR)systems. This paper examined the experience of a hospital with its EHR system in the context of the pandemic. Results showed that the hospital maintains a state-of-the-art health care system to provide quality care to its community and was responsive to the recent crisis. The results were consistent with other comparable hospitals examined in this study. The hospitals were successful in adopting EHR systems. They were able to identify gaps that could be filled with technology add-ons from different software vendors to improve their functionality and thereby provide better \& timely patient care. Managing large volumes of data generated in the normal process of EHR operation and ensuring data privacy and security were the significant challenges faced and are likely to continue in the future.

\section{Keywords: Healthcare Analytics, EHR, Information Systems, Data Analytics, Data Warehouse.}

\section{Introduction}

An Electronic Health Care management system is a collection of information systems for collecting, processing, and distributing health-related data about patients to provide better medical care. They include many subsystems that help hospital administration manage various resources in the care continuum. The point of origin for these systems is creating the Electronic Health Record (EHR). An accumulation of health data is then subjected to various analyses to glean valuable information for decision-making. The Federal government and Medicare are the driving forces behind EHR adoption. They attempt to deal with rising healthcare costs while providing affordable quality health care to patients. They expect that with insights gained from analyzing enormous volumes of data generated by these systems, they will find ways to reduce costs, identify better treatment strategies, extend compassionate care beyond a clinical setting, and make health care equitable to society. This paper illustrates through a few case studies how health care institutions have tried to provide predictive, proactive, and precision care to the communities they serve.

This paper is organized along the following lines. In section two, the field of Business Information Systems is examined to identify the primary building blocks used to construct a health care information system. Section three presents prior case studies of organizations that have employed an aspect of health care information systems. The experiences at the Concord hospital illustrate the actual workflow for a smooth and optimal operation of activities among patients, providers, and administration. Another case study demonstrates the use of telehealth to extend care beyond the doctor's clinic at Blue Ridge Medical group. Finally, to illustrate the use of artificial intelligence in data analytics, Israel's Calit Research Institute's experience is presented. Section four offers the EHR experience at Elegant Health. Section five presents the conclusions.

\section{Contributions from the field of Information Systems}

An Electronic Health Record is a patient record that is initiated in the office of a primary care physician, which gets filled with a primary diagnosis, test results, and treatment recommendations and which may require connection to other information systems such as pharmacy information system, laboratory information system, billing, and insurance information systems. Physicians bear primary responsibility for the accuracy and completeness of the data entered. Therefore, EHRs facilitate the integration of patient health history for planning safe and suitable treatment. When this data is analyzed, effective medicines and therapies for chronic diseases are possible. Healthcare Analytics is the process of analyzing patient data to glean information that could be valuable in making superior decisions in delivering treatments to patients (Kohli \& Tran; 2016); (Amarasingham et al., 2014).

Successful formation of EHRs requires that those who have a stake in the delivery and consumption of health care must contribute to comprehensive EHRs and share relevant data with other stakeholders. The major stakeholders in the EHR landscape are patients who are the direct recipients of benefits, administrators who are the keepers or custodians of health data, and providers who are the hospitals, physicians, and diagnostic agencies. The goals of these three stakeholders are more often in conflict and understanding their concerns and needs must be addressed for the electronic healthcare 


\author{
E-ISSN: 2469-6501 \\ VOL: 7, ISSUE: 8 \\ August/2021 \\ DOI: http://dx.doi.org/10.33642/ijbass.v7n8p7 \\ (c) $\oplus$ \\ https://creativecommons.org/licenses/by/4.0/
}

management system's potential to be fully realized (Kohli \& Tran; 2016).

Information Systems researchers are well prepared to design, develop, and facilitate EHR use in healthcare delivery (Agarwal \& Dhar, 2014). Three foundational concepts are relevant in this context. The first is Enterprise Resource Planning Systems (ERP). The second is Data Integration, a concept in data warehousing. The third is Data Analytics (DA), rooted in statistics, mathematics, and artificial intelligence. These are the three dominant areas that provide a gateway to understanding healthcare analytics. Each one of these abovementioned foundational concepts is elaborated in the following sections.

\subsection{Enterprise Resource Planning Systems (ERP)}

Enterprise resource planning systems are integrated computer programs that manage a company's vital business operations such as finance, accounting, human resources, and customer service. The goal of ERP is to enable easy access to business data and create efficient, streamlined work processes. An effective ERP system allows people to access and update the same information based on permission levels assigned to the system in various organizational units. By sharing data, ERP software enables standardization and streamlining of business processes in a small, locally-based organization or a large multinational organization. ERP systems are developed by software vendors and made available as a complete package suitable for specific industries such as the airline industry, hospitality industry, and other industries, including the healthcare industry. Further, ERP vendors provide customized solutions to business problems and target specific organizations by type and size.

By drawing upon their understanding of IS implementation of business application systems, IS professionals can easily transition to redesign clinical and administrative processes such that disparate pieces of health information get integrated within a health care system (Agarwal \& Dhar, 2014); (Kholi \& Tran, 2016). At the center of an ERP system is the presence of a Data warehouse system.

\subsection{Data Warehousing (DW)}

A data warehouse is a database that stores large volumes of historical data in a form that readily supports data analysis and decision making. In addition to this, a whole new set of technologies and related tools exists to process big data. Big data is a term used to describe data collections that are so enormous, complex, and diverse in formats that traditional data management systems are incapable of dealing with them. Big data relies on database architectures that can store massive volumes of data and process data using parallel processing computer architectures to deliver instantaneous results in formats suited for its users.

IS researchers can develop advanced analytical models using EHR data to identify cost-effective treatments, compare therapies, identify at-risk patients with chronic diseases so that providers can intervene with appropriate treatment (Amarasingham et al., 2014).

\subsection{Data Analytics (DA)}

Analytics is the process of gaining insight from stored data by applying procedures drawn from mathematical, statistical, and artificial intelligence disciplines. The purpose is to drive fact-based decision-making for managing health care issues. Integration of data from various sources is necessary and is the fuel for analytics. Different forms of analytics are possible. Descriptive, Diagnostic, Predictive, and Prescriptive (Amarasingham et al., 2014). Descriptive analytics can answer questions like "What is the demographic distribution of diabetic patients" or what type of patients are readmitted to the hospital within 30 days of discharge? Diagnostic analytics help to answer questions like why something happened "What factors preceded patients who developed diabetes?" predictive analytics can answer the question "What is the likelihood that someone from a particular demographic type will develop diabetes?" Prescriptive analytics can answer the question, "What can a person do to avoid developing a diabetes-related complication." Figure 1. Shows a range of data analytics capabilities that can inform and provide knowledge about the issue at hand and thereby aid providers in identifying effective treatments. See Figure 1.

Figure 1. The Range of capabilities possible using Data Analytics.

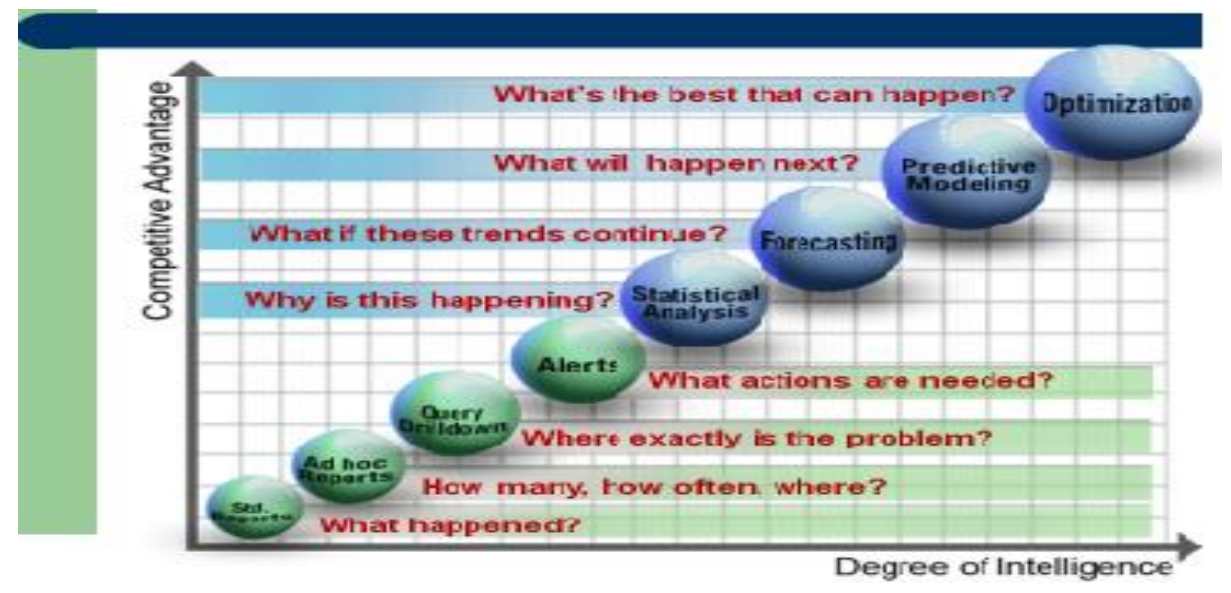




\title{
International Journal of Business and Applied Social Science (IJBASS)
}

\author{
E-ISSN: 2469-6501 \\ VOL: 7, ISSUE: 8 \\ August/2021 \\ DOI: http://dx.doi.org/10.33642/ijbass.v7n8p7 \\ https://creativecommons.org/licenses/by/4.0/
}

\section{Prior Case Studies in Health Care Analytics}

This section presents three hospitals that have successfully adopted EHR. The term EHR in this context encompasses fully functional healthcare information systems with a wide range of data analytics capabilities embedded into their systems. Further, the systems support state-of-the-art network architectures to provide high-speed data transmission capabilities that are reliable, safe, and secure. User-friendly access augment and enhance their systems.

\subsection{Digital Dashboard at Concord Hospital}

In a study recently conducted during the pandemic, Christopher Jason (2020) presents some interesting insights about how EHR systems augment to face the Covid-19 Challenges in a community hospital in New Hampshire. Concord Hospital is an acute-care hospital located in Concord, New Hampshire. It is a charitable organization that exists to meet the health needs of individuals within the community. Concord Hospital, a nonprofit regional health system, provides primary, specialty, and hospital care and is a leader in innovation, quality, safety, and service. The following section describes their experience with a digital dashboard as an addon tool to their EHR.

A digital dashboard is a way of displaying information about critical activities to make effective decisions. In the business world, managers rely on digital dashboards to monitor their scope of work. Similarly, providers and administrators can have a digital dashboard in their hospitals to know the status of their patients and workflow.

Concord hospital serving a small community of people, experienced a situation wherein they found that their EHR was overwhelmed with information. According to Dr. Clarke, the chief medical information officer at Concord, providers had to switch through many screens of information to obtain meaningful data to treat patients. They were searching for an optimizing tool that could augment their EHR and give them the necessary agility to get valuable information (C. Jason, 2020).

During the early stages of the pandemic, Concord hospital faced a new set of challenges, and they had to resort to manual procedures to meet the needs. The hospital had a difficult time finding out how they would track patients, manage their movement within their facility, and determine the COVID status. This function entailed overseeing testing regarding what they are testing for, what is pending, and whether the tests were COVID positive or negative-above all, determining the level of ventilatory support that would be needed.

The hospital found its optimal solution when its EHR vendor provided them with a predictive clinical EHR tool. With this successfully implemented, the physicians saw an immediate decrease in clinician burden. The manual process of monitoring patients, which took them an hour, was completed in fifteen minutes. A more satisfying feeling was discovering that their predictive workflow platform, which was initially intended for everyday situations, became the end-to-end COVID-19 dashboard. With this dashboard, Dr. Clarke remarked, "I can see exactly where the patients were and know what level of ventilatory support they are on." The dashboard eventually ended up being used by their emergency room director in the management of patient movement. The newly implemented optimization tool also supported a notification system which is a class of technologies that facilitates quick updates and universal communication. Having this kind of optimizing tool means that ED doctors receive notifications when a pending test result is returned. Another functionality known as the nurse navigator could notify staff within 48 hours of discharging a patient to take that patient's name off the list and note that a bed was available for new incoming patients.

The Concord hospital uses its EHR dashboard add-on tool beyond the immediate challenges posed by COVID-19. In the context of COPD, which stands for chronic pulmonary obstructive disease, the dashboard's capabilities have gone a long way in managing its census, severity levels, and medications.

Dr. Clarke believes that EHR systems are vast stores of information that, when harnessed with adjunct tools, can augment their inherent functionality. They can become powerful tools at the hands of clinicians, which can genuinely drive better patient care.

\subsection{Telehealth embedded in EHR at Blue Ridge Medical Group}

In another study reported by Bill Siwicki (2020), Blue Ridge Medical group in Blue Ridge, Georgia, resorted to a new virtual-care tool from a health care vendor that allowed a doctor to document directly to the EHR during a telehealth visit. Dr. Dillon Miller, the medical director at this medical group, shares his experience in pivoting to telehealth to meet the challenges erupting from the recent pandemic. The group implemented the AthenaOne app from the vendor Athena Telehealth.

The AthenaOne telehealth system is a HIPAAapproved video conferencing technology that enables a smooth movement from patient scheduling a visit, having a full clinical consult through video conferencing tools, and eventually ending in billing and closing the visit with a followup appointment. This kind of visit is a typical workflow in a doctor's clinic. Through the relaxation of the regulatory hurdles surrounding HIPAA, providers have been able to implement telehealth since the wake of the recent pandemic and find that telehealth is yet another way of adding value to their practice (Siwicki, 2020). According to Dr. Miller, patients were also appreciative as they could receive care from the comfort of their homes. Patients who were old and were too sick to come into the office found this very beneficial.

Telehealth technology aims to deliver an intuitive virtual patient-care experience for both patients and providers that would come close to an "in-office" visit. Physicians can examine patients while updating their electronic health records 


\title{
International Journal of Business and Applied Social Science (IJBASS)
}

\author{
E-ISSN: 2469-6501 \\ VOL: 7, ISSUE: 8 \\ August/2021 \\ DOI: http://dx.doi.org/10.33642/ijbass.v7n8p7 \\ https://creativecommons.org/licenses/by/4.0/
}

in real-time, which means that their health information is updated instantaneously and is not set aside for documenting it later after completing the visit. This procedure helps to maintain the patients' EHR current and complete. It also allows providers to move between appointments quickly and efficiently. At Blue Ridge, scheduling a telehealth visit drove all downstream workflows automatically, integrated with the systems revenue-cycle services, and allowed a seamless billing process.

Having telehealth solutions embedded in the EHR enriched the patient-provider experience and enabled much faster documentation. Telehealth technology has opened doorways for patients to access care in ways that will permanently change care delivery and have demonstrated considerable staying power.

\subsection{Data Analytics at the Calit Research Institute in} Israel

Israel has been at the forefront of coping with the challenges of Covid-19. Laura Lovett (2020) reports about the success of Israel's foremost and largest healthcare organization, The Calit Research Institute. Dr. Ran Baceler, the chief innovation officer at the Calit Research Institute, successfully used a data analytics tool to catch health issues before they become severe. The research institute implemented a system that would provide preventive, predictive, and proactive care to the members of their community. In the wake of the recent COVID-19 pandemic, their healthcare system was able to identify its most vulnerable 200,000 patients to inform them about the spread of the virus and asked them to stay at home. Calit's predictive care system enabled them to take actionable steps and be proactive, and Israel continued to have a low death count relative to other countries.

According to Dr. Ran Bacelor, the Integrated Electronic medical record system was primarily responsible for this. All their patient data were captured in a centralized data warehouse where it was stored and subjected to AI processing techniques. The results from such systems were used to provide predictive, proactive, and preventive care to their population. Their EHR played a critical role and allowed Calit Institute to be proactive.

\section{EHR at Elegant Health.}

Elegant Health is a large nonprofit health care provider on the west coast of the United States that has been in existence for several decades, serving a community of over a million people. Elegant Health's brand of technologically advanced health care service is made possible by a team of dedicated physicians, employees, volunteers, and donors. According to the chief medical officer at Elegant Health, the hospital invested fifty-five million dollars in 2018 for implementing its EHR system. This initiative helped them keep up with the standard practices and become current in health care delivery freeing themselves from the classic paper and chart systems. The vendor of choice was Meditech and its EHR systems. Among the system's many features, the most valuable functionalities were order entry, decision support, and data sharing among patients, providers, and administrators. patient portal, population health management in the community, and financial management were other vital functions provided by the system.

With the implementation of the new system, placing orders for standard medical procedures became easy. Treatment plans were standardized, and relevant procedures were established for the treatments. Further, options to select and order these procedures were available for physicians. Test results could be recorded and made available. The possibility of data sharing enabled physicians, nurses, and administrators to communicate effectively, thereby reducing duplicity, delays, and repetitions. With the Web 2.0 technologies in place, alerts and notifications were sent to the care team members. Physicians could quickly respond to patients and be able to provide timely care.

During the recent pandemic, the EHR system helped them track the COVID-19 trend in the hospital. Their embedded predictive analytics system allowed members in the care team to determine the number of patients admitted and how many tested positive for COVID-19. Patient data was trackable in real-time to help identify patients with co-morbidities, level of severity, ventilator requirements, mortality rates, and patient ethnicity, to name a few.

While the Meditech EHR systems provided almost all the support needed in a hospital facility yet, when it came to practicing telemedicine, the hospital opted to go with another vendor, which the physicians found to be much more rewarding and safer to use and be compliant with HIPAA regulations. The hospital chose Doxy. me for their telemedicine option, which helped them extend care during the challenging times in the pandemic.

Doxy. It is simple and free software and allows physicians to care for their patients outside their clinical settings. By incorporating standard clinical workflows such as patient check-in and waiting room into the design of Doxy. me health care providers and their patients experienced a familiar and natural visit. Patients could enter the virtual office by following a link given by the doctor's office. No special hardware or software was needed. Just any computing device or mobile device with an internet connection was adequate. Doxy. I use state-of-the-art security and encryption protocols to secure data transmission and privacy, thus being consistent with the hospital's overall security standards and architecture. To be true to their mission of providing high-quality, compassionate care to patients, the hospital thus found its answer in Doxy. me that complemented their traditional care delivery at no extra cost to the doctors and patients. Additional charges became applicable to the clinicians only for customizations and branding.

Electronic health records produce, create, interpret, and share immense volumes of data and information. That volume, therefore, means that organizations are at a higher 


\title{
International Journal of Business and Applied Social Science (IJBASS)
}

\author{
E-ISSN: 2469-6501 \\ VOL: 7, ISSUE: 8 \\ August/2021 \\ DOI: http://dx.doi.org/10.33642/ijbass.v7n8p7 \\ (c) (7) \\ https://creativecommons.org/licenses/by/4.0/
}

overall risk for data breaches. Having a large volume of data implies that organizations should develop, follow, and constantly revise a security framework to ensure data security and privacy. Norfleet and Harvey (2020) report that by 2025 approximately $68 \%$ of medical devices will be connected or be connectable to health systems networks, raising a new and complex set of challenges as health systems set out to defend those devices from cyber threats. A severe cyber-attack can bring healthcare providers to a standstill forcing organizations to turn patients away.

In 2019 Elegant Health experienced a security threat issue originating from a physician's office in the vicinity. Although patient data was not compromised and was intact, the hospital recognized that they had to increase their security measures, especially when providers were logging in from remote sites. In response to this threat incident, the hospital invested in a portal system to authenticate and validate their users and improve their network security measures. They moved away from virtual private networks (VPN) and invested in Citrix systems. Citrix is a vendor in network-level security measures.

Traditionally, a virtual private network has been the security solution of choice for providing secure access to corporate systems and files. But VPNs are complex to manage and could introduce network-level security risks. The user experience is less than satisfactory since they are neither scalable nor privacy-friendly compared to the Citrix Workspace systems. Citrix Workspace provides a cloud-based, VPN-less solution to access all intranet web, software as a service (SaaS), mobile, and virtual applications - whether using managed, unmanaged, or bring-your-own devices (BYOD) over any network. Citrix Workspace was preferred as a better choice than VPN to meet security, performance, and scalability requirements for remote access. With this system implemented, the hospital saw better days and quick response times. Ever since then, there have been no security issues. With the appropriate security measures in place, they are confident that they will maintain more than adequate security to their patient data.

According to the chief medical officer at Elegant Health, data management challenges were the most debilitating. The main challenge that Elegant Health experienced was data overload. Enormous volumes of data are generated in the process of providing timely information. "The continuous updates that must be done to bring the system up to date lead to delays and disruptions in day-to-day work," said the medical officer.
To enhance the patient experience, Elegant Health uses a health care management tool called Follow My Health (FMH) which caters to both patients, providers, and administrators. Having this opportunity makes the patientphysician relationship closer than ever. Follow my health is a cloud-based tool that allows patients access to their medical information from a single access point. It is a secure online website that gives patients convenient 24-hour access to personal health information from anywhere with an Internet connection. Using a protected name and password, patients can get health information such as test and lab results, notes from their recent visit to the doctor, future follow-up appointments. Prescriptions ordered, insurance benefits, pharmacies nearby, and literature about their medical conditions can also be obtained.

FMH is a safe and secure environment where patients can communicate privately with their physicians via a secure messaging system. These are just a few of the many options that are available through the system. Providers offer their patients portal access, and patients must register themselves to start using the system. Data about patients then flows directly into their EHR and automatically populates their medical record and becomes available for analysis.

At Elegant Health, while many forms of data analytics were embedded and fully functional, they fell short on optimization tools that rely on artificial intelligence. Optimization modules are on their road map for the following year.

Presently several organizations are using data analytics tools to accelerate Covid-19 drug discovery in addition to other drug discoveries for other diseases. Data analytics tools are being used for contact tracing and when this is combined with data visualization tools the location of Covid-19 cases can be identified. All in all, artificial intelligence-backed analytics is certainly a worthy investment for healthcare institutions.

\section{Conclusion.}

All the above cases mentioned in this paper indicate successful attempts in implementing EHR. And according to the reports of the Chief Information officers in these hospitals, their EHR systems were extensible and adaptable to accommodate add-on modules such as Dash-boards, Telehealth, and optimization modules to make them versatile. The most challenging issue was managing the enormous amounts of data generated by these systems. Such data management challenges lead to data privacy, security, and interoperability issues that need to be addressed to promote the adoption and widespread use of EHR.

\section{References}

Adler-Milstein, J., DesRoches, C.M., Furukawa, M.F., Worzala, C., Charles, D. Kralovec, P. 2014. "More than half of US Hospitals Have at least a basic EHR, but stage 2 criteria remain challenging for most" Health affairs pp1164-1671.

Agarwal, R. and Dhar, V.2014 "Big Data, Data Science and Analytics: The opportunity and challenge for IS Research," Information Systems Research. Pp 443-448. 
Amarasingham, R, Patzer, R.E. Huesch, M., Nguyen, N.Q and Xie, B. 2014. "Implementing Electronic Health Care Predictive Analytics: Considerations and Challenges" Health affairs pp 1148-1154.

Blumenthal, D., and McGraw, D. 2015 "Keeping personal health Information safe: The importance of good data hygiene." Journal of the American Medical Association. P1424.

Figure 1. Analyticsinsight.net. Published in 2013. Downloaded in 2013.

Jason Christopher. (2020). "How a Hospital Optimized its EHR Dashboard to Combat Covid-19". Downloaded from EHRintelligence.com Published on July 2, 2020.

Jercich, Kat. (2020) The Cybersecurity framework organizations should follow to keep data safe. Downloaded from Healthcareitnews.com, published on June 9, 2020.

Kent, Jessica. (2020). How will Big Data Analytics factor into the next phase of COVID-19? Downloaded from Healthitanalytics.com. Published on May 7, 2020.

Kohli, R. \& Tran, S. (2016) "Transforming Healthcare through IS Research" MIS Quarterly, no 40, 2016.

Lovett, L. (2020). "Israel's Calit Research Institute shows that using technology to catch health issues before they become serious." Downloaded from Healthcare Information Management Systems Society Digital (HIMSS20), published on August 25, 2020.

Norfleet Allison \& Marlon Harvey. "Why security is more critical than ever." Downloaded from Healthcare Information Management Systems Society Digital (HIMSS20). Published on May 6, 2020.

Siwicki, Bill. (2020) "How one physician's practice was transformed by EHR-embedded Telehealth." Downloaded from Healthcareitnews.com, published on June 16, 2020. 